\title{
Evolutionary epistemology as a scientific method: a new look upon the units and levels of evolution debate
}

\author{
Nathalie Gontier
}

Received: 1 July 2009/Accepted: 4 November 2009/Published online: 26 May 2010

(C) Springer-Verlag 2010

\begin{abstract}
Evolutionary epistemology can provide a unified scientific methodology that enables scholars to study the evolution of life as well as the evolution of cognition, science, culture and any other phenomenon displayed by living organisms. In this article, three heuristics are provided that allow for a thorough search for the units, levels and mechanisms of evolution. Contrary to previous approaches, units, levels and mechanisms are not identified by pointing out essential features, but rather ostensive definitions are preferred. That is, units are considered as such if a level of evolution and a mechanism of evolution is identifiable. Levels are levels if one can point out units that evolve at that level according to evolutionary mechanisms, and mechanisms are considered as such if one can point out units and levels where the mechanism is active.
\end{abstract}

Keywords Evolutionary epistemology · Units ·

Levels · Evolutionary mechanisms

\section{N. Gontier}

Fund for Science and Technology, Portugal (FCT), Centre for Philosophy of Science, Universidade de Lisboa, Lisbon, Portugal

N. Gontier $(\bowtie)$

Marie Curie Outgoing Research Fellow, Centre for Logic and Philosophy of Science, Vrije Universiteit Brussel,

Pleinlaan 2, 1050 Brussels, Belgium

e-mail: Nathalie.Gontier@vub.ac.be

N. Gontier

Division of Palaeontology, American Museum of Natural

History, New York, NY, USA

\section{Introduction}

Evolutionary epistemology is a branch of naturalized philosophy that originally endorsed the view that both the study of knowledge as a phenomenon as well as the acquirement of knowledge as a scientific activity need to be conducted from within evolutionary theory.

Through the years, the basic tenet (that cognition needs to be studied from within evolutionary theory) has been expanded to include the idea that all behaviours portrayed by living organisms (culture, language, memory, vision, etc.) can be regarded as cognitive processes and that all, therefore, need to be examined in light of evolutionary theory.

In this article, we will argue that evolutionary epistemology can actually provide a unified scientific methodology that enables scholars to study the evolution of life as well as the evolution of cognition, science, culture and any other phenomenon displayed by living organisms.

In order to make this view clear, in what follows, the origin of the field of evolutionary epistemology is sketched, and how evolutionary epistemology relates to the units and levels of selection debate is demonstrated. Second, a more thorough analysis of the units and levels of selection debate is given. Scholars working within both the units and levels of selection debate as well as within evolutionary epistemology have been developing heuristics based on the theory of evolution by means of natural selection to study the evolution of a variety of biological and (traditionally understood to be) extra-biological phenomena such as cognition and culture. As will be demonstrated, in their search for these heuristics, they have actually been developing methodologies, research tools that allow us to study these phenomena.

So far, the heuristics provided have been strongly biased towards the theory of evolution by means of natural 
selection. In this article, we will demonstrate how heuristics can be developed to allow for the inclusion of other evolutionary theories as well.

\section{Evolutionary epistemology}

Evolutionary epistemology is a concept that was first launched by the psychologist Campbell (1974). Intrigued by cognition in general, and in particular with the process of knowledge acquisition in humans as well as in other animals, Campbell (1959, pp. 153-155) wanted to build an 'empirical science of induction', as he called it. More specifically, he wanted to study epistemology (i.e. the philosophical discipline that studies how we come to know) from within evolutionary biology rather than regard it as a distinct branch of philosophy.

In practice, Campbell achieved this goal by pursuing two different goals:

(1) He investigated the different cognitive mechanisms that organisms can portray as outcomes of biological evolution (e.g. how learning by imitation or the ability to have mental representations evolved by means of natural selection).

(2) He investigated how it was possible to apply natural selection not only within the domain of life's evolution, but also within the cognitive, epistemological realm.

The first effort would especially find hearsay in naturalized philosophy where the search for biological foundations of cognition became a widely pursued goal (Gontier 2006). In 1941, Konrad Lorenz already argued that Kant's synthetic a priori claims could, in light of evolution, be reformulated as claims that are ontogenetically a priori and phylogenetically a posteriori. Imprinting or fixed action patterns are to a great extent innate behaviours of individuals but were argued to be somehow acquired throughout the evolution of the species. These ideas were later independently formulated by Campbell as well, to explain why animals appear to have some instinctive knowledge of the world in which they are born prior to experiencing it. In 1963, Karl Popper independently arrived to his evolutionary biology-inspired theory that science progresses along the lines of conjectures (trials) and refutations (errors). In his book entitled Objective Knowledge, he too would use the term evolutionary epistemology (Popper 1975, p. 67). Toulmin (1972) would argue that science can be regarded as a selective process; and Skinner (1981, 1986), the founding father of behaviourism or instructionism as it is nowadays sometimes called, assessed that trial and error learning or operant conditioning are similar to selectionist processes. Eventually, all these events would inspire Bradie (1986) to distinguish between two programs: the evolutionary epistemology of cognitive mechanisms (EEM) and the evolutionary epistemology of scientific theories (EET). The normative EEM program studied cognition as an outcome of evolution by means of natural selection; it is the '...attempt to extend evolutionary theory to the explanation of the development of cognitive structures...' (Bradie 1986, p. 403). The other, descriptive EET program studied science and the question of scientific progress by analogy to selectionist processes; it is 'the attempt to analyze the growth of knowledge using evolutionary models, drawn from biology...' (Bradie 1986, p. 403).

It is especially the second point that Campbell made, which is of interest for the purpose of this article. His investigation of how exactly it is possible to apply natural selection not only to the evolution of life but also to the products of life's evolution-which is a question of how natural selection operates-would find hearsay in both biology and philosophy of biology. More specifically, his examination of how exactly it is that natural selection operates run juxtaposed with the units and levels of selection debate.

Any attempt to understand how selection operates and how it can be expanded to include non-biological phenomena is ultimately a quest of specifying where, at what levels it operates, and on what units. The Modern Synthesis straightforwardly argues that evolution by means of natural selection takes place at the interface between a phenotype and the environment; it does not make statements on how to apply natural selection to phenomena such as the evolution of cognition, creative thinking, science or culture. In order to overcome this obstacle, Campbell (1959) abstracted a template from natural selection which he first dubbed 'blind variation and selective survival' and later reformulated into 'blind variation and selective retention' (Campbell 1960). This template can be regarded as a formula with heuristic potential: regardless of the phenomenon under study, if we can understand that phenomenon to vary blindly and be selectively retained, then this phenomenon evolves by means of natural selection (i.e. it is a unit of selection), and, importantly, this phenomenon also operates as a selective process.

The abstraction of a template (of the operation) of natural selection to be able to apply it to other domains besides the evolution of life was also independently achieved by Lewontin (1970). He argued that it was possible to abstract a 'logical skeleton' of natural selection that is a 'powerful predictive system for changes at all levels of biological organisation'. The skeleton consists of the following 'three principles': '1. Different individuals in a population have different morphologies, physiologies, and behaviours (phenotypic variation). 2. Different phenotypes have different rates of survival and reproduction in different 
environments (differential fitness). 3. There is a correlation between parents and offspring in the contribution of each to future generations (fitness is heritable). These three principles embody the principle of evolution by natural selection' (Lewontin 1970, p. 1).

Formulas that provide us with heuristic information of how natural selection operates were later also formulated by Hull (1980, 1981; Hull et al. 2001) who introduced the 'replication, variation and environmental interaction' scheme; and Plotkin (1994) who argued for a 'generate, test, regenerate' formula that focussed on the 'replicator, interactor and the lineage'.

These schemes not merely inform us on the operation of natural selection (how it works), they also inform us on the nature of the units of selection and the levels at which selection operates (what they are). It is exactly because of this that there is a strong interrelation with the terminology put forward by scholars who tried to untangle the units and levels of selection and those that wanted to provide heuristics of the operation of natural selection.

\section{A closer look at the units and levels of selection debate}

The debate over the units and levels of selection started in biology with the introduction of Wynne-Edwards' (1962) book on group selection. The main point of discussion revolved around whether or not the fact that a group sometimes benefits from the presence of certain traits in the individuals that make up the group (e.g. altruistic or selfsacrificing behaviour) can actually account for the positive selection of these traits, even though these traits can have negative effects on the fitness of the individuals that posses them. Wynne-Edwards claimed that certain traits might indeed be for the good of the group (i.e. contribute to the overall fitness of the group) even though it disregards the individual's fitness or even if the trait is maladaptive to the individual.

Williams (1966) strongly opposed such a view, arguing on the contrary that only the gene can be the ultimate beneficiary of selection. Even though it might appear that groups benefit from the positive selection of some traits over others, that selection process can always be explained by and reduced to the gene. Following Williams, Richard Dawkins would further argue that for something to be considered a unit of selection, it must be a replicator, i.e. 'an entity in the universe of which copies are made' (Dawkins 1982 , p. 162), and this replicator must posses longevity, fecundity and copying-fidelity (Dawkins 1976, p. 19). The rationale behind these requirements was 'that an entity must have a low rate of spontaneous, endogenous change, if the selective advantage of its phenotypic effects over those of rival ('allelic') entities is to have any significant effect'
(Dawkins 1982, p. 164). He would conclude that only genes, and perhaps memes, can fulfil these properties and that, therefore, they are the true and exclusive units of selection. Individuals or groups are but 'clouds in the sky' or 'duststorms in the desert' (Dawkins 1999, pp. 99-100) because they lack the stability and integrity required for copying. Rather than understanding organisms or phenotypes as the unit of selection, as was subscribed to by Mayr and other founders of the Modern Synthesis, Dawkins (1983) reduced the role of the organism and of populations to mere vehicles that house the true units of selection. Dawkins (1982, p. 162) states: '...[t]here are two ways in which we can characterize natural selection. Both are correct: they simply focus on different aspects of the same process. Evolution results from the differential survival of replicators. Genes are replicators; organisms and groups of organisms are not replicators, they are vehicles in which replicators travel about. Vehicle selection is the process by which some vehicles are more successful than other vehicles in ensuring the survival of their replicators'.

In his Extended phenotype, Dawkins (1999, p. 114) would further argue that there is a nested hierarchy of levels at which selection takes place, but every time, this selection process can be reduced to and explained by the selection of replicators.

Especially Dawkins' definition of a unit of selection triggered great controversy over how exactly a unit of selection can be defined; it was at this point in time that philosophers of evolutionary biology in general, and evolutionary epistemologists in specific entered the debate. They started analysing the conceptual issues over units of selection, and they have been doing so ever since.

To start with, Hull $(1980,1981)$ argued that the 'vehicle selection' that Dawkins was talking about might be better understood as the selection of 'interactors'; interactors being any 'entity that directly interacts in a cohesive whole with its environment in such a way that replication is differential' (Hull 1980, p. 318). "When Dawkins defines 'replicator', he has replicators interacting with their environment in two ways - to produce copies of them and to influence their own survival and the survival of their copies. Just as Dawkins coined the term 'replicator' for the entities that function in the first process, I have suggested 'interactor' for the entities that function in the second process" (Hull 1980, p. 318).

Following this characterization, Hull (1980, p. 318) could abstract his own logical skeleton of selection, and he defined selection as 'a process in which the differential extinction and proliferation of interactors cause the differential perpetuation of the replicators that produced them'.

Hull (1980, pp. 320-327) further emphasized that this characterization of the selection process makes it clear that 
there are different levels of selection: levels where replication occurs (he pointed out the genetic and organismal level), and levels where interaction occurs that results in differential replication (an example that he gave was populations).

Wimsatt (1981, p. 124) would also criticize Williams and Dawkins' claim that selection at any level can and must always be reduced to gene selection because only this entity is stable and hereditary. Wimsatt (1981, p. 127) would, on the contrary, argue in favour of 'higher-level biological units of selection' that can fulfil the 'structural requirements' of being hereditary, portraying variance in comparison to other such units, and, therefore, having differential fitness values (Wimsatt 1981, pp. 165-166). He would subsequently define units based upon this variance in fitness they can portray at a certain level. More specifically, a unit of selection is: ' . ...any entity for which there is heritable context-independent variance in fitness among entities at that level which does not appear as heritable context-independent variance in fitness (and thus, for which the variance in fitness is context-dependent) at any lower level of organization' (Wimsatt 1981, p. 144).

The fact that this definition of a unit of selection again is interrelated with the definition of a level would make Brandon (1982) call for a separate study of levels of selection. Following Wimsatt, Brandon would also define levels in close relation to fitness and adaptedness (Brandon 1982, p. 315). Brandon would furthermore define natural selection as '...the differential reproduction of biological entities which is due to the differential adaptedness of those entities to a common environment' (Brandon 1982, p. 318).

In the years to come, the replicator-interactor dichotomy would by and large set the stage for all theorizing on the units and levels of natural selection, and these concepts have indeed allowed for the identification of a large number of units and levels. Nonetheless, from the second half of the 1980s onwards, scholars would begin to see the flaws inherent to this dichotomy. Genic pluralists, as they would call themselves (Kitcher et al. 1990, pp. 159-160; Sterelny and Kitcher 1988; Waters 1991, p. 555), would criticize Dawkins' and Williams' move to reduce selection to the level of the gene. They argue instead that the gene itself can be the target of selection at many different levels of biological organisation. As such, genes are considered to be interactors (Lloyd 2005, 3.4). On the other hand, developmental systems theorists (Griffiths and Gray 1994, pp. 292-295; 1997; Oyama et al. 2001) would argue that whole developmental systems (e.g. the hormone system or the vascular system) or even life cycles can be considered as units of selection even though they are not replicated from one generation to the next. Moreover, developmental systems theories and genic pluralism provide opposing views because the former argue that selection is possible at a number of different entities even though they are not replicated from one generation to the next, while genic pluralists argue that only the gene is the target of selection, albeit at many levels (for a discussion see Sterelny et al. 1996).

Griesemer (2000) would try and combine these opposing ideas by introducing the notion of a 'reproducer' as the unit of selection. Although systems are not replicated, Griesemer argues that such systems are somehow reproduced form one generation to the next and as such these 'reproducers' can be considered as units of selection. More specifically; 'Populations of reproducers have the capacity to evolve, insofar as the pieces of development that realize their reproductive capacities themselves have heritable properties that vary. For reproducers at a given level to be units of evolution, their developmental component parts must also be reproducers' (Griesemer 2000, p. 363).

Griesemer further argues that reproducers can account for and integrate replicators: 'Replication is inheritance with coding mechanisms of development. Inheritance is reproduction with evolved mechanisms of development. Since replication processes are reproduction processes, they must also satisfy the material overlap requirement for reproduction' (Griesemer 2000, p. 366).

In sum, rather than choosing between replicators or interactors as the true units of selection, today scholars are arguing more and more in favour of unit pluralism. Moreover, they are also coming to terms with level pluralism, that is, they are investigating what exactly the consequences are of accepting multilevel selection (Maynard Smith and Szatzmáry 1995; Okasha 2005; Vrba and Eldredge 1984).

\section{Moving beyond selectionist inspired approaches for identifying units and levels}

So far, units and levels have always been defined from within the theory of evolution by means of natural selection. That is, until now it has always been taken for granted that natural selection is the evolutionary mechanism according to which units evolve at certain levels. Depending on how natural selection is defined or argued to operate, scholars have argued that for something to be a unit of selection, this unit must portray (additive) variation; be transmittable (heritable, replicatable or reproducible); demonstrate (emergent) adaptation or the capacity to adapt; have differential fitness values (that are in turn heritable or transmittable); etc. (Dawkins 1976; Lewontin 1970; Griesemer 2000; Sober 1984, p. 204; Vrba 1989; Eldredge 1985; Vrba and Gould 1986; Wimsatt 1980).

In other words, the criteria used to include or exclude something from being a level or a unit is theory-dependent 
if not biased towards the theory of evolution by means of natural selection.

Moreover, the above-listed criteria that units or levels must fulfil to be considered as such are in a real sense comprehended to be inherent, if not intrinsic properties of these units and levels. It is, therefore, not at all surprising that Hull $(1980,1981)$ characterized the quest for units and levels as a metaphysical endeavour (and see also Kitcher et al. 1990, p. 159).

Current evolutionary biology, however, is making it clear that we need to try and move beyond the metaphysical issues as well as allow for the inclusion of evolutionary theories other than natural selection in regard to the identification of levels and units: We need to do so for the following two reasons. For one, natural selection, although an important evolutionary mechanism, is not the exclusive mechanism according to which life or the products of life evolve. Indeed, a number of complementary and alternative evolutionary mechanisms have been identified such as symbiogenesis, hybridization, niche construction, drift, the ratchet effect, Baldwin effect, ${ }^{1}$ etc. Stated otherwise, adhering to an evolutionary view makes it necessary to endorse that if a certain natural phenomenon is unexplainable by natural selection, then the alternative can only be that it has to be explainable by another evolutionary mechanism. It simply cannot be that it did not evolve just because it did not evolve by means of natural selection. Secondly, and following from the previous point, units and levels can and must be identifiable without making use of the theory of evolution by means of natural selection. That is, evolutionary mechanisms are always active on some units at certain levels, even though the latter are not replicators or interactors. It is, therefore, better to endorse a more neutral approach and move from the units and levels of selection debate to the units and levels of evolution debate.

In this regard, scholars working within the field of symbiogenesis have already put forward the symbiome (Sapp 2004, p. 1047) and symbiont (Gontier 2007, pp. 174-175) as units of evolution - and the following logical skeleton of symbiogenesis has already been abstracted: 'Universal symbiogenesis is the process whereby new entities are introduced because of the interactions between (different) previously independently existing entities. These interactions encompass horizontal mergings and the new entities that emerge because of this are called symbionts. The process is irreversible and discontinuous' (Gontier 2007, pp. 174-175).

\footnotetext{
1 The Baldwin and ratchet effect can to a certain extent be comprehended from within selectionist approaches, yet certain aspects of these mechanisms extend and complement mere selectionist views.
}

In other words, the time has come to not only recognize unit pluralism, and multilevel selection, but we need also to accept the consequences of mechanism pluralism and the fact that this introduces units and levels of evolution that do not necessarily have the intrinsic properties demanded of replicators, interactors, reproducers, etc.

In what follows, a pragmatic and extensional way to identify units and levels of evolution as well as evolutionary mechanisms is, therefore, favoured over a metaphysical one.

\section{Heuristics to respectively study units, levels and mechanisms of evolution}

Given that there is such a thing as evolution, one needs to specify the units, levels and evolutionary mechanisms by which this evolution occurs. This can be done by following the heuristics set out in Tables 1, 2 and 3.

General remarks on the heuristics

It should be noted from the beginning that it is not regarded as meaningful to a priori speculate upon what evolution itself is, what is essential to it, or what form any one of its units, levels and evolutionary mechanisms can take on. No (essential) definitions are provided. Rather, units, levels and evolutionary mechanisms are defined extensional.

More specifically, in the heuristics it is investigated whether features, traits, events, elements, etc. that are assumed $^{2}$ to be relevant for evolution (from now on designated as $x$ ), are a unit, level or evolutionary mechanism that is indeed involved in evolution. If it can be demonstrated that $x$ is at minimum one of the latter three (a unit, level or mechanism), then $x$ is a part of (an extension of) evolution.

What form a unit of evolution takes on, what relates all units or what characterizes all units is not defined beforehand. Rather, an $x$ (an element, trait, etc.) that is assumed to be a unit of evolution, is recognized as such if and only if at least one level can be pointed out where $x$ is a unit, and at least one evolutionary mechanism can be pointed out to which $x$ is subjected. A unit of evolution can only be a unit if it evolves at a certain level; if it evolves at a certain level, it is subjected to a certain evolutionary mechanism. Thus, it cannot be the case that an element is shown to evolve, without this evolution taking place at a certain level. Or it cannot be shown that a unit is present at a certain level, without it being subjected to a certain evolutionary mechanism at that level.

The extensional definition of a unit of evolution, therefore, is that which evolves at a certain evolution level and

\footnotetext{
${ }^{2}$ Either intuitively or experimentally.
} 
Table 1 Is $x$ (a feature, trait, space, event, element, etc., that is presumed relevant for evolution) a unit in/of evolution? (read from left to right and top-down)

\begin{tabular}{|c|c|c|c|}
\hline$?$ & \multicolumn{3}{|c|}{ Try to prove that it is a unit of evolution (1 example suffices). Thus go to yes } \\
\hline \multirow{7}{*}{$\begin{array}{l}\mathrm{Y} \\
\mathrm{E} \\
\mathrm{S}\end{array}$} & \multirow{2}{*}{$\begin{array}{l}\text { Where? At which } \\
\text { level is } x \text { the subject of } \\
\text { evolution }\end{array}$} & \multicolumn{2}{|c|}{ Not one level found? $\mathrm{X}$ is not a unit, go to no } \\
\hline & & $\begin{array}{l}\text { One/multiple level(s)? } \\
\text { Identify them all. (Justifies } \\
\text { that } \mathrm{x} \text { is a unit.) }\end{array}$ & $\begin{array}{l}\text { Via which evolutionary } \\
\text { mechanism(s)? Identify } \\
\text { them all }\end{array}$ \\
\hline & Since when? & \multicolumn{2}{|c|}{$\begin{array}{l}\text { When did } \mathrm{x} \text { first originate in time and when did it become a } \\
\text { unit of evolution? }\end{array}$} \\
\hline & $\begin{array}{l}\text { How does this unit } \mathrm{x} \\
\text { interact with other }\end{array}$ & \multicolumn{2}{|c|}{$\begin{array}{l}\text { Can this unit be divided into one or several subunits? } \\
\text { If so, then are they also units in evolution? }\end{array}$} \\
\hline & units? & \multicolumn{2}{|c|}{$\begin{array}{l}\text { Can this unit be absorbed into one or several superunits? If } \\
\text { so, then are they also units in evolution? }\end{array}$} \\
\hline & $\begin{array}{l}\text { Can this unit also be } \\
\text { regarded as a level } \\
\text { and/or mechanism of } \\
\text { evolution? }\end{array}$ & \multicolumn{2}{|c|}{$\begin{array}{l}\text { ? \& yes: try and treat the unit as a level and/or a } \\
\text { mechanism, thus go to level and/or mechanism }\end{array}$} \\
\hline & Relevance? & \multicolumn{2}{|c|}{ Is the unit $\mathrm{x}$ sufficient and/or necessary for evolution? } \\
\hline \multirow{2}{*}{$\begin{array}{l}\mathrm{N} \\
\mathrm{O}\end{array}$} & \multirow{2}{*}{$\begin{array}{l}\text { Level and/or } \\
\text { mechanism? }\end{array}$} & \multicolumn{2}{|c|}{ ? or Yes: go to level and/or mechanism } \\
\hline & & \multicolumn{2}{|c|}{$\begin{array}{l}\text { No: treat } \mathrm{x} \text { as irrelevant for evolution until proven } \\
\text { otherwise }\end{array}$} \\
\hline
\end{tabular}

Table 2 Is $x$ (a feature, trait, space, event, element, etc., that is presumed relevant for evolution) a level in/of evolution? (read from left to right and top-down)

\begin{tabular}{|c|c|c|}
\hline$?$ & \multicolumn{2}{|c|}{ Try to prove that it is a level of evolution (1 example suffices). Thus go to yes } \\
\hline $\mathrm{Y}$ & \multirow{2}{*}{$\begin{array}{l}\text { How many/which units } \\
\text { evolve at this level? }\end{array}$} & Not one unit, $\mathrm{x}$ is not a level of evolution, go to no \\
\hline $\begin{array}{l}E \\
S\end{array}$ & & $\begin{array}{l}\text { One/multiple unit(s)? Identify them all. (Justifies that } \mathrm{x} \text { is a } \\
\text { level.) }\end{array}$ \\
\hline & $\begin{array}{l}\text { How many } \\
\text { evolutionary } \\
\text { mechanisms are active } \\
\text { at (not on) this level? }\end{array}$ & $\begin{array}{l}\text { Equals the question: how many evolutionary mechanisms } \\
\text { are active upon the units that evolve at this level. (testing } \\
\text { device) }\end{array}$ \\
\hline & $\begin{array}{l}\text { What is the ontological } \\
\text { status of the level? }\end{array}$ & $\begin{array}{l}\text { The level is an abstract notion that facilitates theory } \\
\text { formation/ an exiting entity }\end{array}$ \\
\hline & Since when? & $\begin{array}{l}\text { Locate the origin of } \mathrm{x} \text { in time or when it becomes necessary } \\
\text { to invoke } \mathrm{x} \text { as an abstract notion in the theory of evolution }\end{array}$ \\
\hline & \multirow{2}{*}{$\begin{array}{l}\text { How does this level } \mathrm{x} \\
\text { interact with other } \\
\text { levels? }\end{array}$} & $\begin{array}{l}\text { Can this level be divided into sublevels? If so, are they also } \\
\text { levels in evolution? }\end{array}$ \\
\hline & & $\begin{array}{l}\text { Can this level be absorbed into superlevels? If so, are they } \\
\text { also levels in evolution? }\end{array}$ \\
\hline & $\begin{array}{l}\text { Can this level also be } \\
\text { regarded as a unit } \\
\text { and/or mechanism of } \\
\text { evolution? }\end{array}$ & $\begin{array}{l}\text { ? \& yes: try and treat the level as a unit and/or mechanism, } \\
\text { thus go to unit and/or mechanism }\end{array}$ \\
\hline & Relevance? & Is the level x sufficient and/or necessary for evolution? \\
\hline \multirow{2}{*}{$\begin{array}{l}\mathrm{N} \\
\mathrm{O}\end{array}$} & \multirow{2}{*}{$\begin{array}{l}\text { Unit and/or } \\
\text { mechanism? }\end{array}$} & ? or Yes: go to unit and/or mechanism \\
\hline & & $\begin{array}{l}\text { No: treat } \mathrm{x} \text { as irrelevant for evolution until proven } \\
\text { otherwise }\end{array}$ \\
\hline
\end{tabular}

is subjected to a certain evolutionary mechanism is a unit of evolution.

Whether $x$ (a space, feature, trait, event, element, etc.) is recognized as a level of evolution depends on whether it is possible to identify at least one unit of evolution that is subjected to minimally one evolutionary mechanism at this level, and at least one evolutionary mechanism needs to be active $a t$ the level (not on the level). The latter (that at least 
Table 3 Is $x$ (an evolutionary mechanism, feature, trait, space, event, element, etc., that is presumed relevant for evolution) an evolutionary mechanism involved in/on evolution? (read from left to right and top-down)

\begin{tabular}{|c|c|c|}
\hline ? & \multicolumn{2}{|c|}{ Try to prove that $\mathrm{x}$ is an evolutionary mechanism involved in evolution. Thus go to yes } \\
\hline & \multirow{2}{*}{$\begin{array}{l}\text { On how many units is } \\
\text { this } \quad \text { evolutionary } \\
\text { mechanism working? }\end{array}$} & $\begin{array}{l}\text { Not one unit: } \mathrm{x} \text { is not an evolutionary mechanism involved in } \\
\text { evolution }\end{array}$ \\
\hline & & $\begin{array}{l}\text { One/miltiple unit(s). Identify them all. (Justifies that } \mathrm{x} \text { is an } \\
\text { evolutionary mechanism involved in evolution.) }\end{array}$ \\
\hline & $\begin{array}{l}\text { At (not on) how many } \\
\text { levels of evolution is this } \\
\text { evolutionary mechanism } \\
\text { active? }\end{array}$ & $\begin{array}{l}\text { Equals the question: the units that are subjected to this } \\
\text { evolutionary mechanism, at how many levels are they subjected } \\
\text { to it? }\end{array}$ \\
\hline & \multicolumn{2}{|c|}{$\begin{array}{l}\text { How does the mechanism work? Which conditions need to be met in order for the } \\
\text { evolutionary mechanism to occur? Answer requires (universal) EE formulas of the } \\
\text { workings of the mechanism }\end{array}$} \\
\hline & Since when? & $\begin{array}{l}\text { Locate in time when these conditions are met regarding each } \\
\text { unit and each level = when the evolutionary mechanism became } \\
\text { a mechanism involved in evolution at that unit and/or level }\end{array}$ \\
\hline & \multirow[t]{2}{*}{$\begin{array}{l}\text { How does this } \\
\text { mechanism } \mathrm{x} \text { interact } \\
\text { with other mechanisms? }\end{array}$} & $\begin{array}{l}\text { Can this mechanism be divided into sub-mechanism(s)? } \\
\text { (Depends on the presence of subconditions.) If so, are they also } \\
\text { mechanisms of evolution? }\end{array}$ \\
\hline & & $\begin{array}{l}\text { Can this mechanism be absorbed into a super-mechanism(s)? } \\
\text { (Depends on the existence of a mechanism that allows to } \\
\text { combine different mechanisms into one single mechanism.) If } \\
\text { so, are they also mechanisms of evolution? }\end{array}$ \\
\hline & $\begin{array}{l}\text { Can this mechanism also } \\
\text { be regarded as a unit } \\
\text { and/or level of evolution? }\end{array}$ & $\begin{array}{l}\text { ? \& yes: try and treat the mechanism as a unit and/or level, thus } \\
\text { go to unit and/or level }\end{array}$ \\
\hline & Relevance? & $\mathrm{x}$ sumclent and/or \\
\hline \multirow{2}{*}{$\begin{array}{l}\mathrm{N} \\
\mathrm{O}\end{array}$} & \multirow[t]{2}{*}{ Unit and/or level? } & ? or Yes: go to $\mathbf{u}$ \\
\hline & & No: treat $\mathrm{x}$ as irrelevant for evolution un \\
\hline
\end{tabular}

one evolutionary mechanism needs to be active at the level) is automatically the case if a unit of evolution has been identified to evolve at $x$. Thus, that $x$ is a level of evolution is proven by identifying one unit of evolution that is subjected to at least one evolutionary mechanism at $x$. Regarding evolutionary mechanisms, $x$ is recognized as an evolutionary mechanism if $x$ at least works on one unit of evolution, that evolves at a certain level of evolution. Or, stated more elaborately, $x$ is an evolutionary mechanism involved in evolution if at least one unit of evolution is found to be subjected to it, and at least one level of evolution is found where $x$ is active at (not on). But again, the latter is automatically the case (that one level of evolution is found where the mechanism is active at), if at least one unit is found to be subjected to the mechanism, because a unit is always subjected to a mechanism at a certain level.

This is a circular way of denoting units, levels and evolutionary mechanisms because it brings the identification of units, levels and evolutionary mechanisms into the system. The identification of anyone involves pointing out, quite ostensively, the presence of the other two. What units, levels and mechanisms are is not defined from outside the system.
Although circular, this is actually a rather strong as well as neutral and pragmatic way of searching for units, levels and evolutionary mechanisms. It is strong because all the three elements need to be identified. It is neutral because it neither a priori specifies the nature of $x$, nor the nature of a unit, level or mechanism. Defining a unit beforehand (as, e.g. a replicator or an interactor) might set clear boundaries to what is and what is not a unit, but it might also lead to the exclusion of a trait even though it is subjected to an evolutionary mechanism at a certain level of evolution.

Finally, because there are no rigorous definitions given, the identification of $x$ as a unit does not exclude it to possibly also be a level or perhaps even a mechanism. The same also goes for a level and a mechanism.

\section{Walking through the heuristics}

Suppose then that we have an $x$ that is presumed to be relevant for evolution. $X$ is relevant for evolution if and only if it can be proven to be at minimum either a unit, level or mechanism of evolution.

In what follows, it is explained how we can know that $x$ is or is not a unit, level or mechanism. 
How to examine whether $x$ is a unit of evolution

How do we know that $x$ (e.g. a gene, meme, replicator, reproducer, symbiont, symbiome, chromosome, phenotype, trait, behaviour, system, pattern, organism, etc.) is a unit of evolution (Table 1)?

The question mark phase

If we do not know without examination of $x$ that $x$ is a unit of evolution, then we can evidently neither exclude it from being one, nor recognize it as a unit. In such an uncertain situation, the heuristic recommends that we try to prove that it is a unit of evolution. One example suffices to demonstrate that it is a unit. It is useless to try and prove that it is not a unit of evolution (in such an uncertain situation) for the following reason. If $x$ is not a unit, then the heuristic says that we need to try and prove whether it is a level or an evolutionary mechanism. But even if one identifies $x$ as a level or a mechanism, this will not have proven that $x$ is not a unit. This is the case because being a unit does not a priori exclude something from being a possible level or perhaps even a mechanism. ${ }^{3}$

How then can we prove that $x$ is a unit? $X$ is a unit of evolution if it can be proven that $x$ is subjected to a certain evolutionary mechanism, at a certain level of evolution. If it is subjected to a mechanism, then a level must be identifiable because a unit always evolves at a certain level. (An example would be that the organism is a unit of evolution that evolves at the level of the environment by means of natural selection.)

The first question that, therefore, needs to be raised if one wants to prove that $x$ is a unit is: where, at which level is it a unit?

If one or several such levels of evolution are identified where $x$ is subjected to a certain evolutionary mechanism, then this proves that $x$ is a unit of evolution. If, on the other hand, not even one level is found where $x$ is the subject of evolution, $x$ is not a unit of evolution. In this case, one should go to no phase.

The yes phase

The yes phase is distinguishable into an identification, question constraining and generating, and an evaluation phase.

\footnotetext{
$\overline{3}$ That a unit of evolution might also be a mechanism of evolution sounds strange, but given the multiplicity of the evolutionary mechanisms that exist, and given the fact that we do not know what a unit can be, this, nevertheless, needs to be a possibility that is taken into consideration.
}

The identification phase

If one skipped the question mark phase, and immediately argued that $x$ is a unit of evolution, then such action can only be justified if again one or several levels are identified where $x$ is the subject of evolution. In other words, a small test is built into the heuristic that allows one to demonstrate that $x$ is indeed a unit of evolution. It furthermore needs to be identified according to which evolutionary mecha$n i s m(s) x$ evolves at that single or at those multiple levels. In other words, the heuristic allows for the testing of two ideas. The first is that there is such as thing as multilevel evolution (a more commonly held view nowadays, e.g. Okasha 2005); the second (not so commonly subscribed to idea) is that there can simultaneously be different evolutionary mechanisms active upon the same unit.

If the unit is subjected to several mechanisms at one level, then the relation or possible interaction between the evolutionary mechanisms needs to be explained in regard to this unit. Furthermore, it needs to be explained how the same unit can simultaneously be subjected to different mechanisms at the same level.

If the unit is subjected to the same mechanism at multiple levels, then it needs to be explained how the same mechanism can be active on the same unit at all these levels. If the unit is subjected to different mechanisms at multiple levels, then it needs to be explained how different mechanisms can be active on the same unit at different levels and also the interaction between these mechanisms in regard to this unit needs to be examined.

Finally, if a unit is the subject of evolution at multiple levels, then it also needs to be explained how these levels interact or relate to one another, specifically in regard to this unit.

The answers to these latter questions will be of an evolutionary epistemological nature. A consequence of the recognition of several levels is that the same unit can evolve at several levels. Like Russian dolls, genes, for example, can be the target of selection at the level of the phenotype (e.g. regarding their expression) and/or at the level of the environment (via the phenotype in which they are expressed). The question then becomes how these levels interact in regard to this unit and how the same unit can be subjected to the same evolutionary mechanism at several levels.

A consequence of the recognition of several mechanisms is that these can act upon (parts of) the same unit simultaneously, at the same or different levels. If this is the case, then it becomes a possibility that the mechanisms interact with one another, and even if they do not, it needs to be explained why they do not interact.

The topic of the existence or (im)possibility of interactions between evolutionary mechanisms is by and large neglected in the evolutionary epistemological literature. It is 
mostly implicitly assumed that if one evolutionary mechanism is active, then all other evolutionary mechanisms are not. It is counterintuitive to ask whether an evolutionary mechanism itself can be subjected to other evolutionary mechanisms (e.g. can natural selection be subjected to symbiogenesis; or can symbiogenesis be the target of selection?), and it is counterintuitive to ask whether evolutionary mechanisms can, for example, be divided into submechanisms, or whether they can be classified hierarchically, into supermechanisms. It will be a future challenge for evolutionary epistemologists to frame the questions as well as answers in regard to how evolutionary mechanisms relate to one another. However, these questions need to be raised simply because several evolutionary mechanisms exist (I return to this during the discussion of evolutionary mechanisms).

Having proven or justified that $x$ is a unit of evolution at minimally one level, where it is subjected to minimally one evolutionary mechanism sums up the identification phase and allows us to move on to the phase of generation and constraining of research questions.

\section{Question-generating and constraining phase}

When all the above questions are answered, or if there is only one level where $x$ is subjected to one evolutionary mechanism, then one has identified $x$ as a unit of evolution. At this point, the heuristic goes beyond the mere identification, mapping or categorizing of units. On the contrary, it provides you with questions that allow you to systematically investigate how $x$ has evolved. The heuristic is, therefore, also a question-generating, investigative tool. The first question that is generated is the since when question.

Having proven that $x$ is a unit of evolution, it becomes necessary to ask whether $x$ from its origin onwards has always been a unit of evolution. This can be done by first locating the origin of $x$ in time, and secondly by examining whether this date coincides with the origin of $x$ as a unit of evolution for each level where it is a unit. In other words, one should consider the theoretical possibility that $x$ has prior existence to biological evolution, but that this $x$ can nevertheless become involved in the evolution of life (it might have a prior physical or chemical existence; nucleotides and amino acids precede the origin of life).

The following question is: how does this unit $x$ interact with other units in general? More specifically, can the unit be divided into several subunits, that are also units in evolution in their own right? Or can this unit be absorbed into one or more superunits, that in turn are also units of evolution? Units are best regarded as Matruskas, structures that are both decomposable into different substructures and that can at the same time be grouped into larger superstructures (genes can be divided into nucleotides, sugar bases, and phosphates, or introns and exons, while they can be grouped into RNA, DNA, chromosomes, etc). In this regard, the heuristic is also a unit-detecting device. Asking about the possibility to distinguish subunits and superunits will expose new units of evolution that in turn need to be investigated independently, from unit onwards.

Another query is whether the unit $x$ can also be regarded as either a level or a mechanism of evolution. Especially when the unit can be divided into subunits, and when the unit itself is thus a superunit, the latter might be a level as well. Here too, the same criteria define levels and mechanisms: if something is a level, then it means that units can be identified as well as evolutionary mechanisms that are active upon those units, at those levels. Mechanisms are only mechanisms if they are active upon units at levels. If uncertain, then the test begins from (respectively) level and mechanism onwards.

So far, the identification of $x$ as a unit as well as the questions that arise from this identification, have allowed one to generate as well as constrain specific research avenues.

\section{Evaluation phase}

Now, it is demonstrated how the heuristic also introduces a series of questions that allows one to evaluate the information that is obtained by investigating $x$ as a unit; as well as which role this information can play in a unifying theory on the origin and evolution of life.

More specifically, we can investigate how relevant the unit $x$ is or has been in evolution by asking whether the unit is sufficient and/or necessary for life or its products to evolve. These questions are not raised to investigate what is 'essential' to evolution. Rather, they serve to evaluate the importance of the unit. They allow us to get an overview of the results that have been achieved by examining this unit as well as the study that still needs to be done to solve the problem of the evolution of life and its products. Suppose, for example, that there exists an all-encompassing Language Acquisition Device (module/gene, Chomsky 1965; Fodor 1983) that is a unit of evolution. Examining this LAD from within the above heuristic (as well as all its subunits-if there are such subunits) will for the most part have solved the problem of how language evolved. If on the other hand, the human-specific shape and position of the tongue has been demonstrated to be a unit of evolution, then this will leave the researcher with the task of examining numerous other units, before he will try solving the problem at hand.

If the unit $x$ is sufficient for evolution to occur, then it needs to be explained why there are other units involved in evolution (if there are). It seems likely that there does not 
exist such a single unit $x$, but this nonetheless needs to be proven. If it is not sufficient, then it needs to be explained where it falls short in explaining evolution.

If the unit $x$ is necessary for evolution, then $x$ needs to be treated as a general (and perhaps even universal) unit of evolution. This means that the unit $x$ must have been present, at least during a specific period of time, during life's evolution, and, important from an epistemological perspective, every theory that tries to explain the evolution of life needs to take into account the evolution of this unit. If $x$ is not necessary for evolution to occur, then neither of the two points are required. Rather, the unit may be regarded as peculiar to a certain kind or aspect of evolution (e.g. the evolution of the mind, or of language, or of mammals), and it must thus not necessarily form part of a general theory on the evolution of life.

The no phase

If $x$ is not a unit of evolution, then one needs to investigate whether $x$ is either a mechanism or a level of evolution. If it is either a level or a mechanism, then it needs to be treated accordingly, from within the heuristics presented in Tables 2 and 3. If it is neither a level or a mechanism, then $x$ should be treated as irrelevant for evolution until proven otherwise.

\section{How to examine whether $x$ is a level of evolution}

How do we know that $x$ (e.g. the environment, the brain, culture, etc.) is or is not a level of evolution (Table 2)?

The question mark phase

If we do not know whether $x$ is a level of evolution, then how do we begin examining $x$ ?

In such an uncertain situation, the heuristic recommends that we try and prove that $x$ is a level of evolution. Just as was the case with units of evolution, it is not enough to try and prove the no path and thus to prove that $x$ is a unit or mechanism, because at least units (and perhaps even evolutionary mechanisms) can also be levels of evolution. An organism can itself be unit of evolution, but it also houses genes that are possibly units of evolution. In this regard, the organism becomes a level where these genes can evolve.

How then do we prove that $x$ is a level of evolution? $X$ is a level of evolution if one or several units of evolution can be demonstrated to be the subject of one or several evolutionary mechanisms at that level $x$. Identifying one or several units of evolution that evolve at $x$, therefore, justifies that $x$ is a level of evolution. The main question that is raised in this regard is therefore, how many units can be distinguished to evolve at level $x$ ? Every additionally identified unit confirms that $x$ is indeed a level.
If not even one unit of evolution can be identified to evolve at $x$, then $x$ is not a level of evolution, and one can, therefore, immediately go to no phase.

The yes phase

The yes phase is again distinguishable as an identification, question-constraining and generating, and an evaluation phase.

\section{Identification phase}

If one immediately chose the yes scenario, then this action can be justified if one or several units of evolution can be identified to evolve at this level. If the latter is indeed the case, then the goal is to identify all units that are the subject of evolution at this level. Every additional unit that is recognized to evolve at this level warrants the claim that $x$ is a level.

If multiple units are indeed identified, then it also needs to be specified how these units interact or relate to one another, specifically in regard to this level.

At this point, a testing device is built into the heuristic, by asking how many evolutionary mechanisms are active at this level, not on this level.

Especially the latter point is important. It would be illegitimate to recursively ask the same questions raised regarding the study of units of evolution and simply replace the word 'unit' with the word 'level'. That is, it would be wrong to ask 'How did/does this level evolve? Identify the evolutionary mechanisms by which the level evolved in regard to (for) this unit'.

This question is illegitimate because the existence of the level does not depend on the existence of the unit. The level did not evolve in regard to or for the unit. This would be teleological. The environment, for example, did not evolve for the phenotype, ${ }^{4}$ or culture did not evolve for pointing to occur.

What does make sense is to ask like this: since when did this level become a level in the evolution of the unit? Since when is a unit subjected to an evolutionary mechanism at this level? But this brings us to the since when question, and so the above question between brackets becomes unnecessary.

What also makes sense is to ask how the level itself evolved. However, if it evolved-which in turn means that it is subjected to one or several evolutionary mechanism(s) at certain level(s) - then one needs to treat the level as a

\footnotetext{
4 The environment evolves differently because of the emergence of the phenotype (as, e.g. demonstrated by niche construction, Lewontin 2000), but it did not evolve 'for' the phenotype before the latter was present.
} 
possible unit of evolution and thus start from unit onwards. Here too, the above question between brackets thus becomes unnecessary.

It is, therefore, only intelligible to ask how many evolutionary mechanisms are active at this level (rather than on this level). This question in turn equals the question: how many evolutionary mechanisms are active upon the units that evolve at this level. The identification of evolutionary mechanisms that are active at this level thus depends upon the units that are the subject of evolution at this level. These units should in principal have been identified during the previous step of the heuristic. And the evolutionary mechanisms that are active upon these units should in principle already be known.

By and large, the identification of the evolutionary mechanisms that are active at this level $x$ on one or more units, is thus a test that allows to confirm the results that were obtained by following the rules set forth by the previous step of the heuristic. The recognition of one mechanism that is active at this level on a unit again confirms and even justifies that $x$ is a level. But again, it is advised to independently expose all evolutionary mechanisms that are active at this level. (A simple count of the mechanisms that are active at the level can also provide insight into the nature of the level.)

If multiple mechanisms are indeed identified to be active, then a further question that needs to be asked is how these evolutionary mechanisms interact or relate to one another regarding this level in particular. How does the ratchet effect for example interact with the Baldwin effect at the cultural level in regard to the different units that are exposed to these mechanisms?

\section{Question-generating and constraining phase}

Once $x$ has been identified as a level, the heuristic again generates new and specific research questions, as was the case with the identification of $x$ as a unit.

Specific to the level heuristic is that it asks the following question: what is the ontological status of the level. If something is a unit of evolution, then it exists, otherwise it cannot be the target of a certain evolutionary mechanism. Genes, for example are, under certain circumstances, units of natural selection. The concept of a gene however is a theoretical, abstract notion (Stadler et al. 2009). That is, when a certain group of molecules (nucleotides, phosphates, sugar molecules, etc.) is structured in a certain manner, it is called a gene. When it is said that a gene is the target of selection, one actually argues that it is this whole group of molecules that is subjected to selection. The term 'gene' is just an abbreviation for this group of molecules, a handy concept that does not make it necessary to sum up all the different elements that make up a gene. But even though the gene is an abstract notion, the things it denotes are actual, existing entities.

Contrary to units, the ontological status of levels is not always clairvoyant. Assuming for example that culture or the community are levels of evolution (an assumption that still needs to be justified), the ontological status of these levels is uncertain. Does culture exist independently from the individuals that lie at its emergence (does it form a superorganic structure that has existence prior to and outside individuals as e.g. Kroeber 1923 thought); or is 'culture' merely an abstract notion that is introduced to facilitate theory formation (e.g. Sapir 1917)? Does there exist a 'language community' (Chomsky 1965; de Saussure 1972; Wittgenstein 1953) without individual language-bearing organisms?

Thus, it becomes necessary to identify the ontological status of the level, whether it is an abstract notion that facilitates theory formation, or whether it is an existing entity in the world.

The question that asks about the ontological status of the level needs to be raised to be able to answer the questions raised by the next step in the heuristic, namely the since when-step.

Having demonstrated that $x$ is a level of evolution, it becomes necessary to investigate since when $x$ is a level of evolution.

More specifically, if $x$ is an existing entity, then it can have an existence prior to it being a level of the evolution of life and its products. If it is indeed an existing entity, then the origin of $x$, therefore, needs to be examined independently, as well as when exactly $x$ became a level of life's evolution, or one of life's products, for each unit where it serves as a level. Earth for example exists from 4.5 billion years ago, but only becomes a level in the evolution of life from 3.85 billion years ago-which is the estimated time that life first arose on earth. And homologues of Broca's region already exist in the monkey brain (the F5 region, Fadiga et al. 2000), but it is unclear when they became a level of language evolution.

If $x$ is not an existing entity, then it needs to be demonstrated when it becomes necessary to invoke $x$ as an abstract notion in the theory on evolution; as well as when it becomes necessary to invoke $x$ as an abstract notion for each unit where it serves as a level.

When $x$ first became a level of evolution (abstract or real) depends on the age of the unit(s) that is/are identified to be subjected to (an) evolutionary mechanism(s) at that level. The age of the oldest unit that evolves/(d) at that level equals the age that $x$ became a level of evolution. In order to determine this date, it needs to be dated when each unit that evolves at this level, became a unit of evolution at that level.

Similar to the unit heuristic, the following steps of this heuristic allows one to examine how this level interacts 
with other levels. Moreover, as was the case with the units of evolution, it is interesting to ask whether this level can be divided into sub- or superlevels of evolution. The level of the environment might be absorbed into large categories such as 'the physical world' or subdivided into different landscapes, niches, etc. The community level might for example be absorbed into the cultural level or divided into the family level, the peer level, the educational level, etc. If there are indeed such sub-or superlevels, then these levels need to be studied in their own right from levels of evolution onwards. In this regard the heuristic is also a leveldetecting device.

Not only is it interesting to try and identify other levels by studying this level $x$ in particular, one also needs to try and identify the level under examination as a possible unit or a mechanism of evolution.

That the level $x$ is also a unit of evolution depends on whether it is itself subjected to evolutionary mechanisms that are involved in evolution at certain (other) levels of evolution. (The ontological status of the level might provide a possible break-in to such investigation.) If this is indeed the case, then the level should be treated as a unit from unit onwards. In this regard again, the heuristic can be a unit-detecting device. It can also be a mechanismdetecting device. In order to investigate whether a level is also a mechanism, the level $x$ must be demonstrated to work on units at other levels (see also the discussion of mechanisms later in the text).

\section{Evaluation phase}

If $x$ is indeed a level of evolution, then the final step of the heuristic again entails specifying how relevant the level is or has been, in the evolution of life. Again this is not required so that one can detect what is essential to evolution. Rather, this will help evaluate the importance of the results obtained when studying this level $x$, and it will shed light upon what still needs to be dealt with in the future if one wants to unravel the evolution of life.

The relevance of the level can be deliberated by specifying whether $x$ as a level is sufficient or necessary for evolution, or both.

If $x$ as a level (e.g. the environment) is indeed sufficient for life to evolve, then it should be explained why other levels (e.g. species) are involved in the evolution of life (if indeed there are). If it is not sufficient, then it should be explained where it falls short.

If $x$ as a level is necessary for evolution to occur, then $x$ should be treated as a general (universal) level of evolution that needs to be present during a certain time in evolution. In this scenario, $x$ should also be accounted for in all theories that deal with the evolution of life.
If it is not necessary, then $x$ can be treated as a peculiarity that neither necessarily is present during a certain time in evolution, nor need it necessarily be part of a theory that deals with the evolution of life or aspects thereof.

The no phase

If $x$ is not a level of evolution, then one needs to investigate whether it is a unit or mechanism in evolution. If neither of the latter can be proven, then $x$ can be treated as irrelevant for the evolution of life, until proven otherwise.

How to examine whether $x$ is an evolutionary mechanism involved in evolution

If we have an $x$ (e.g. natural selection, symbiogenesis, drift, the ratchet effect, the Baldwin effect, niche construction, etc.) of which we do not know whether it is an evolutionary mechanism or not, then how do we begin examining whether this $x$ is an evolutionary mechanism?

The question mark phase

If we are not sure, then we need to try and prove that it is indeed an evolutionary mechanism and thus to go to the yes phase. One can proof that $x$ is a evolutionary mechanism by identifying at least one unit upon which the presumed evolutionary mechanism $x$ is active. If not one unit of evolution is found to be subjected to $x$, then the latter is not an evolutionary mechanism.

If one or several such units are indeed identified upon which $x$ is active, then it is justified to call $x$ an evolutionary mechanism.

The yes phase

Again, the yes phase in distinguishable into an identification, question constraining and generating, and an evaluation phase.

\section{Identification phase}

If one immediately went to the yes-phase of the heuristic, then this action can only be justified if at least one unit of evolution can be identified that is subjected to this evolutionary mechanism.

At this stage, again all units upon which the mechanism is active need to be identified; thus, the how many-question prevails again.

If amongst the identified units that are subjected to this evolutionary mechanism are newly identified evolution units, then the latter need to be investigated separately, 
from unit onwards. Once more, the heuristic can, therefore, identify new units of evolution.

If multiple units have indeed been identified, then it also needs to be examined how these units interact or relate to one another, specifically in regard to this evolutionary mechanism.

Next step is to identify at (not on) how many levels of evolution this mechanism is active. This question equals the question at how many levels the identified units of evolution are subjected to this evolutionary mechanism. Thus, in principle, the levels are already identified during the study of the units. Nonetheless, the heuristic advises to sum up the levels independently. This allows to have an independent notion of the amount of levels at which the mechanism is active and it enables one to again test the previous step. One or more levels again confirm that it is justified to identify $x$ as an mechanism.

If multiple levels are identified, then it needs to be examined how these different levels interact or relate to one another, specifically in regard to this mechanism.

\section{Question-generating and constraining phase}

Having identified $x$ as an evolutionary mechanism, the heuristic again offers questions that allow for a more systematic examination of the mechanism.

The following question is specific to the examination of evolutionary mechanisms: How does the mechanism work? More specifically, which conditions need to be met for the evolutionary mechanism to occur?

Evolutionary mechanisms are not constant forces that are always present. Rather, they only occur when certain conditions are met. These conditions are constant ${ }^{5}$ and always need to be met, before an evolutionary mechanism becomes active.

The answer to the question, which conditions need to be met, will again be of an evolutionary epistemological nature. More specifically, the answer will involve one or more universal formulas that are abstracted from the evolutionary mechanism that explains the workings of the mechanism. The evolutionary epistemological formulas have presently only been abstracted from two evolutionary mechanisms: natural selection (e.g. Campbell's 1974 'blind variation and selective retention', Plotkin' 1994 'generate, test, regenerate' formula) and symbiogenesis (Gontier 2007). As is well known in the evolutionary epistemological community, such formulas are themselves heuristics that inform us upon the workings of the mechanisms.

\footnotetext{
5 Which does not necessarily imply that the mechanism itself is constantly active. However, when it is active, the same conditions must be present.
}

These formulas will also explain equivalent questions such as 'How is it possible that the same evolutionary mechanism is active on one or different units, at the same or different levels?' According to Campbell (1997), natural selection, for example, can be active upon one or multiple units at the same or different levels, as long as all these units vary blindly, and as long as all these levels allow for the selective retention of these units.

It will be a challenge for the future to abstract universal formulas from all other known evolutionary mechanisms. Such abstractions or 'logical skeletons' (Lewontin 1970) that allow to identify the conditions that need to be met for a certain evolutionary mechanism to become active are highly necessary for future theory formation (Gontier 2008).

In evolution studies, these conditions need to be specified to be able to answer the following question of the heuristic: Since when did $x$ become a mechanism involved in the evolution of life, for the units it is active upon and the levels it is active at.

This question can be answered as soon as one can date when the mechanism became active upon each unit and at each level of evolution where it is known to be active. But how do we know when did it become active upon these units and at these levels?

We can investigate when the mechanism became involved in evolution on a certain unit by examining when the conditions are met regarding this unit (e.g. in the case of natural selection, when a unit became a replicator or interactor).

We can investigate when the mechanism became involved in evolution at a certain level of evolution by examining when the conditions are met regarding this level of evolution (e.g. in the case of natural selection, when a level of evolution achieved the ability to selectively retain replicators). The latter, therefore, is again dependable on the units that evolve at this level.

The next step that the heuristic entails is in asking how the mechanism $x$ interacts with other mechanisms that are known to be involved in the evolution of life. This is evidently done by comparing the mechanism $x$ with all other mechanisms that are known to be involved in evolution. Although perhaps counterintuitive, the mere existence of different mechanisms makes it at least theoretically possible that these mechanisms interact in one form or another, by, for example, competing or being cooperatively or complementarily active upon certain units. These theoretical possibilities need to be demonstrated to exist, or it needs to be explained why they cannot occur in real life. Nonetheless, they need to be the subject of future investigation.

Again it also becomes interesting to ask whether the mechanism can be divided into submechanisms or supermechanisms. If so, then these sub- and supermechanisms need to be studied from mechanism onwards, and it needs 
to be investigated whether they are also involved in evolution in their own right.

That submechanisms exist depends upon the existence of subconditions that can be regarded as single, independent mechanisms (e.g. is the ratchet effect or the Baldwin effect a submechanism of natural selection or a mechanism in its own right?). The existence of supermechanisms will depend on the existence of mechanisms that allow to combine different mechanisms in a yet to be defined manner (perhaps hierarchically).

The discussion of sub- and supermechanisms is also strange if not counterintuitive. However, given that the evolutionary epistemological formulas (itself heuristics) of most evolutionary mechanisms still need to be abstracted, the possibility of such sub- and supermechanisms cannot be excluded a priori.

Next, the heuristic advises us to investigate whether the mechanism can also be regarded either as a unit or a level of evolution. Thus, once more, the heuristic generates as well as constrains research questions. The former would entail that the mechanism is itself subjected to at least one evolutionary mechanism at, at least, one level of evolution. The latter would entail that at least one unit of evolution can be demonstrated to evolve according to an identified evolutionary mechanism at this mechanism $x$, or that an evolutionary mechanism is active upon a unit of evolution at this mechanism.

It is currently unknown how mechanisms can themselves be the subject of evolution (as units), or how they can be levels where (certain types of) evolution occurs. Although a theoretical consideration that cannot a priori be proven to be wrong (for it depends on the nature of the evolutionary mechanisms), the validity of the above two questions remains to be proven.

\section{Evaluation phase}

Finally, here too an evaluation phase is built into the heuristic to enable one to investigate the importance and relevance of the mechanism in the evolution of life as well as the role it needs to play in the theorizing on life's evolution and its products. This will again allow for an evaluation of the results obtained when studying this mechanism. Supposing, for example, that $x$ is a mechanism that is identified to be active on all known units, at all known levels, the mechanism will for a great deal have solved the puzzle of how life evolved.

The relevance is tested by examining whether the evolutionary mechanism $x$ is sufficient for evolution to occur or not, and whether it is necessary for evolution to occur or not.

If sufficient, then it needs to be explained why other mechanisms are involved in the evolution of life (if there are). If necessary, then $x$ needs to be treated as an evolutionary mechanism that needs to be active at some point during evolution and that, therefore, needs to be accounted for in all theories on the evolution of life.

If unnecessary, then the evolutionary mechanism can be treated as a peculiar/particular mechanism that does not need to be active during life's evolution and that does not necessarily need to be accounted for in all theories on the evolution of life (the inference of the mechanism $x$ in a theory will depend on which aspects of evolution are highlighted in that theory).

\section{The no phase}

If $x$ is not an evolutionary mechanism, then it needs to be investigated whether it is either a unit or a level of evolution. If neither, then $x$ is irrelevant for the evolution of life until proven otherwise (depending on incoming data).

\section{Conclusions and future research}

The original goal of evolutionary epistemology was to study cognition like any other biological phenomenon, but the field has come a long way ever since. Together with biologists that were interested in the units and levels of selection, evolutionary epistemology has set the stage for the implementation of evolutionary, selectionist thinking in many different scientific domains, ranging from neural Darwinism to memetics, immunology, economics, developmental systems theory and so on (Cziko 1995).

This wide application has allowed us to currently identify a new series of problems, unforeseen by the original thinkers that applied their insights only to the evolution of the phenotype and cognition. More specifically, when studying physical traits, a (set of) genes, a (set of) behaviours, developmental systems, cultural units, artefacts, neural maps, cognitive traits, altruistic rules etc., it is not always clear what kind of units they are (interactors, replicators or reproducers) of what kinds of evolution, and at what levels they evolve-whether indeed they are elements of evolution, and even if we were to identify them as either one, it still then does not guaranty us that it will help us solve the problem of how these units actually evolved, according to which mechanisms and at what levels.

Moreover, complex phenomena like the evolution of culture or language seem to involve a multiplicity of units (e.g. the supralaryngeal vocal tract, Broca and Wernicke's area, social behaviour), levels (the cultural level, biological level, genetic level) and mechanisms (natural selection, the Baldwin and ratchet effect).

Coming to terms with evolution as something that always occurs, realizing that there can be different kinds of 
evolution (the evolution of life, culture, the brain), as well as the recognition of a multiplicity of units, levels and mechanisms made us realize that we now need to start an active search for the different kinds of evolution as well as the units, levels and mechanisms in their own right. The time is now ripe to move beyond the metaphysical, on natural selection-based and biased views of what the units and levels are or ought to be that do the evolving. Besides unit and level pluralism, also mechanism pluralism must be endorsed.

Evolutionary epistemology can be the method that allows us to start this active search for units, levels and mechanism of evolution. Rather than search for intrinsic properties of units, levels and evolutionary mechanisms, here a pragmatic approach is favoured. $X$ is a unit if it evolves at a level according to a certain mechanism; $x$ is a level if certain units evolve at $x$ because they are subjected to certain mechanisms; $x$ is a mechanism if it is active upon certain units at certain levels. Not one step in the heuristic is taken for granted-not even the units, levels or evolutionary mechanisms, since the existence of any one of them depends upon the existence of the other two.

The heuristics set forward in this article are not merely devices that categorize the current data on evolution. Rather, these are the genuine search engines that allow identifying the different units, levels and mechanisms of evolution. The heuristics furthermore systematize, generate, constrain and allow one to evaluate different research avenues.

The above listed heuristics also makes us realize that an incredible amount of work needs to be done if we are ever to develop a unified theory of evolution. Indeed, this is a work that can succeed only if scholars unite. Databases need to be built, which group the identified units, levels, mechanisms and kinds of evolution. Moreover, logical skeletons need to be built, which list the conditions that need to be fulfilled for an evolutionary mechanism to be active.

It is amazing how simple the different questions can be framed, and how difficult it is to provide an answer to every single one of them. It, therefore, needs to be stressed that every single question that is raised by the heuristic regarding a certain $x$ that might be involved in evolution, involves a research project on its own. Nonetheless, all these different research routes can be brought together into one all encompassing research plan. At the onset, the focus should lie at subunits, sublevels and submechanisms and the more knowledge we have of these, the more we can build 'the big picture' by investigating superunits, superlevels and supermechanisms and their relations.

The above heuristic presents a methodology that will allow us to firmly ground, test as well as identify the units, levels and mechanisms of evolution. EE will systematize, synthesize and further analyse the current theories and debates into a unifying science of evolution.

Acknowledgements My warm thanks are extended to Olga Pombo for inviting me to organise the Lisbon conference. I am also very grateful for the help I got in this endeavour from my co-organizers Helena Abreu, Francisco Carrapiço, André Levy and Marco Pina. I acknowledge the financial support received from the Portuguese Fund for Science and Technology (the FACC). I am indebted to all contributors for making the volumes what they are. Finally, I want to sincerely thank the editors-in-chief of Theory in Biosciences for providing us with a good home for our proceedings.

\section{References}

Bradie M (1986) Assessing evolutionary epistemology. Biol Philos 1:401-459

Brandon RN (1982) The levels of selection. In: Brandon RN, Burian RM (eds) (1984) Genes, organisms, populations. MIT, Cambridge, pp 133-139

Campbell DT (1959) Methodological suggestions from a comparative psychology of knowledge processes. Inquiry 2(3):152-183

Campbell DT (1960) Blind variation and selective retention in creative thought as in other knowledge processes. Psychol Rev 67:380-400

Campbell DT (1974) Evolutionary epistemology. In: Schlipp PA (ed) The philosophy of Karl Popper, vol I. La Salle, Chicago, pp 413459

Campbell DT (1997) From evolutionary epistemology via selection theory to a sociology of scientific validity. Evol Cogn 3:5-38

Chomsky N (1965) Aspects of the theory of syntax. MIT, Cambridge

Cziko G (1995) Without miracles. MIT Press, Cambridge

Dawkins R (1976) The selfish gene. Oxford University Press, New York

Dawkins R (1982) Replicators and vehicles. In: Brandon NR, Burian RM (eds) (1984) Genes, organisms, populations. MIT, Cambridge, pp 161-179

Dawkins R (1983) Universal Darwinism. In: Hull DL, Ruse M (eds) The philosophy of biology. Oxford University Press, New York, pp 15-35

Dawkins R (1999/1982) The extended replicator. Oxford University Press, New York

de Saussure F (1972) Cours de linguistique générale. Editions Payot, Paris

Eldredge N (1985) Unfinished synthesis. Oxford University Press, New York

Fadiga L et al (2000) Visuomotor neurons: ambiguity of the discharge or 'motor' perception. Int J Psychophysiol 35:165-177

Fodor J (1983) Modularity in the mind. MIT, Cambridge

Gontier N (2006) Evolutionary epistemology. The Internet encyclopaedia of philosophy. http://www.iep.utm.edu/e/evo-epis.htm

Gontier N (2007) Universal symbiogenesis: an alternative to universal selectionist accounts of evolution. Symbiosis 44:167-181

Gontier N (2008) The dynamics of language activity: an evolutionary reconstruction. Unpublished $\mathrm{PhD}$ dissertation, Vrije Universiteit Brussel, Brussels

Griesemer J (2000) Development, culture and the units of inheritance. Philos Sci 67(Proceedings):S348-S368

Griffiths PE, Gray RD (1994) Developmental systems and evolutionary explanation. J Philos XCI(6):277-304

Griffiths PE, Gray RD (1997) Replicator II: judgement day. Biol Philos 12(4):471-492 
Hull DL (1980) Individuality and selection. Annu Rev Ecol Syst 11:311-332

Hull DL (1981) Units of evolution: a metaphysical essay. In: Jensen UJ, Harré R (eds) Studies in the concept of evolution. The Harvester Press, London, pp 23-44

Hull DL, Langman RE, Glenn S (2001) A general account of selection: biology, immunology, and behaviour. Behav Brain Sci 24:511-573

Kitcher P, Sterelny K, Waters CK (1990) The illusory riches of Sober's monism. J Philos 87(3):158-161

Kroeber AL (1923) Anthropology. Harbinger Books, New York

Lewontin R (1970) The levels of selection. Annu Rev Ecol Syst 1:1-18

Lewontin R (2000) The triple helix. Harvard University Press, Cambridge

Lloyd E (2005) Units and levels of selection. The Stanford encyclopaedia of philosophy. http://plato.stanford.edu/entries/ selection-units/\#3.3

Lorenz K (1941) Kants Lehre vom Apriorischen im Lichte gegenwärtiger Biologie. Blätter für Deutsche Philos 15:94-125

Maynard Smith J, Szathmáry E (1995) The major transitions in evolution. Oxford University Press, New York

Okasha S (2005) Multilevel selection and the major transitions in evolution. Philos Sci 72:1013-1025

Oyama S, Griffiths PE, Gray RD (2001) Cycles of contingency. MIT Press, Cambridge

Plotkin H (1994) Darwin machines and the nature of knowledge. Penguin Books, London

Popper K (1963) Conjectures and refutations. Routledge \& Kegan Paul, London

Popper K (1975) Objective knowledge, and evolutionary approach. Oxford University Press, London (reprinted with corrections)

Sapir E (1917) Do we need a superorganic? Am Anthropol 19: $441-447$

Sapp J (2004) The dynamics of symbiosis: an historical overview. Can J Bot 82:1046-1056
Skinner BF (1981) Selection by consequences. Science 213:501-504 Skinner BF (1986) The evolution of verbal behaviour. J Exp Anal Behav 45:115-122

Sober E (1984) The nature of selection. MIT Press, Cambridge

Stadler P, Prohaska S, Forst C, Krakauer D (2009) Defining genes: a computational framework. Theory Biosci 128(3):165-170. doi: 10.1007/s12064-009-0067-y

Sterelny K, Kitcher P (1988) The return of the gene. J Philos 85: 339-361

Sterelny K, Smith KC, Dickison M (1996) The extended replicator. Biol Philos 11(3):377-403

Toulmin S (1972) Human understanding. Princeton University Press, Princeton

Vrba E (1989) Levels of selection and sorting with special reference to the species level. Oxf Surv Evol Biol 6:111-168

Vrba E, Eldredge N (1984) Individuals, hierarchies and processes: towards a more complete evolutionary theory. Palaeobiology 10:146-171

Vrba E, Gould SJ (1986) The hierarchical expansion of sorting and selection: sorting and selection cannot be equated. Palaeobiology $12: 217-228$

Waters CK (1991) Tempered realism about the force of selection. Philos Sci 58(4):553-573

Williams GC (1966) Adaptation and natural selection. Princeton University Press, Princeton

Wimsatt WC (1980) Reductionistic research strategies and their biases in the units of selection controversy. In: Nickles $\mathrm{T}$ (ed) Scientific discovery, vol II. Reidel, Dordrecht, pp 213-259

Wimsatt WC (1981) The units of selection and the structure of the multi-level genome. In: Asquith PD, Giere RN (eds) PSA. Philosophy of Science Association, East Lansing, pp 122-183

Wittgenstein L (1953) Philosophical investigations, translated by Anscombe GEM. Basil Blackwell, Oxford

Wynne-Edwards VC (1962) Animal dispersion in relation to social behavior. Oliver \& Boyd, London 